\title{
Pressure effect on micellization of non-ionic surfactant Triton X-100
}

Yanis R. Espinosa, Ernesto R. Caffarena, Yanina Berrueta Martínez, and J. Raúl Grigera

Citation: The Journal of Chemical Physics 148, 074901 (2018); doi: 10.1063/1.5003358

View online: https://doi.org/10.1063/1.5003358

View Table of Contents: http://aip.scitation.org/toc/jcp/148/7

Published by the American Institute of Physics

AP| $\begin{aligned} & \text { The Journal of } \\ & \text { Chemical Physics }\end{aligned}$ PERSPECTIVES 


\title{
Pressure effect on micellization of non-ionic surfactant Triton X-100
}

\author{
Yanis R. Espinosa, ${ }^{1}$ Ernesto R. Caffarena, ${ }^{2, a)}$ Yanina Berrueta Martínez, ${ }^{3}$ and J. Raúl Grigera ${ }^{4}$ \\ ${ }^{1}$ Instituto de Física de Líquidos y Sistemas Biológicos (CONICET-UNLP), Calle 59 Nro 789, \\ B1900BTE La Plata, Argentina \\ ${ }^{2}$ Programa de Computação Científica (PROCC), Fundação Oswaldo Cruz. Manguinhos, \\ CEP 21040-360 Rio de Janeiro, Brazil \\ ${ }^{3}$ Universidad de La Plata, 47 y 115, B1900 La Plata, Argentina \\ ${ }^{4}$ CEQUINOR, Universidad de La Plata and CONICET, 47 y 115, B1900 La Plata, Argentina
}

(Received 5 September 2017; accepted 1 February 2018; published online 20 February 2018)

\begin{abstract}
Micellar aggregates can be arranged in new types of conformational assemblies when they are isotropically compressed. Thus, the pressure effects in the underlying fundamental interactions leading to self-assembly of micellar aggregates can be represented by changes in the phase boundaries with increasing pressure. In this paper, we have employed molecular dynamics simulations to study the self-assembly of micelles composed of the non-ionic surfactant Triton X-100 at the atomic scale, monitoring the changes in the solvation dynamics when the micelles are subjected to a wide range of hydrostatic pressures. The computational molecular model was capable of self-assembling and forming a non-ionic micelle, which subsequently was coupled to a high-pressure barostat producing a geometric transition of the micelle due to changes in the solvation dynamics. Accordingly, under a high pressure regime, the hydrogen bonds are redistributed, the water density is modified, and water acts as an unstructured liquid, capable of penetrating into the micelle. Published by AIP Publishing. https://doi.org/10.1063/1.5003358
\end{abstract}

\section{INTRODUCTION}

Micelles can be regarded as a biological molecular prototype whose geometric shape can be self-assembled from amphiphilic surfactants. ${ }^{1}$ The structure, function, thermodynamics, and conformational dynamics of these systems are usually studied using thermal perturbation, pressure, or by the addition of inorganic salts and organic additives. ${ }^{2-6}$

A particular interest in these systems lies in their highpressure behavior. Unlike proteins, whose structure can be lost under the effect of the pressure enhancement, micellar aggregates do not denature when they are isotropically compressed. Furthermore, new types of conformational assemblies can be arranged in some cases. ${ }^{7,8}$

A particular issue that remains under discussion is the volume change under high pressures. In agreement with Le Chatelier's principle, pressure augmentation is accompanied by a negative volume change in the system. ${ }^{9}$ Furthermore, in some cases, the volume change $(\Delta \mathrm{V})$ magnitude can be minuscule or even positive with increasing pressure. ${ }^{10,11}$ Therefore, the real challenge is the precise quantification of different parameters involved in the change volume, i.e., hydration, hydrophobic interaction, cavities, or electrostriction. ${ }^{1,6,12-14}$

Thus, in micellar systems, the pressure effects in the underlying fundamental interactions can be represented by changes in the phase boundaries with increasing pressure. Thus, qualitative descriptors, such as variations in solvation, critical micelle concentration, and aggregation number, have

a)Author to whom correspondence should be addressed: ernesto.caffarena@ fiocruz.br been taken into account aiming at the understanding of the hydrophobic stability, leading to the self-assembly of the surfactant and its pressure dependence. . $^{7,8,15}$

At the beginning of the $1960 \mathrm{~s}$, studies pioneered by Hamann, ${ }^{16}$ about the influence of pressure on the micelles in aqueous solutions, started to be accomplished, wherein the critical micelle concentration (CMC) was determined for the first time for each increase in hydrostatic pressure. By measuring the specific conductivity of the solutions as a function of the sodium dodecyl sulfate (SDS) molality, it was observed that the CMC initially increases with increasing pressure. ${ }^{16}$ Later on, Kaneshina and co-workers analyzed the formation of sodium alkyl sulfates (ionic surfactants) and hexaoxy ethylene dodecyl ether $\left(\mathrm{C}_{12} \mathrm{E}_{6}\right)$ (nonionic surfactants) micelles by measuring conductivity ${ }^{17}$ and laser light-scattering. ${ }^{18}$ They observed that for ionic surfactants the initial compression under pressures close to $100 \mathrm{MPa}$ provoked an increase in the monomeric concentration, causing a redistribution of the surfactant molecules in the micelles into smaller subunits. Also, the authors showed that a higher compression ( $>150 \mathrm{MPa}$ ) promotes both a decrease in the monomeric concentration and the formation of larger micelles. However, the aggregation number of the resulting $\mathrm{C}_{12} \mathrm{E}_{6}$ micelles decreased at the same time that its concentration increased with the consequent heightening of pressure due to the increased hydration of oxyethylene groups in $\mathrm{C}_{12} \mathrm{E}_{6}$.

Subsequently, Tanaka et al. ${ }^{19}$ determined the solubility limit of ionic surfactants with pressure and Offen ${ }^{20}$ proposed a transformation from micelles to the hydrated solid state. Additionally, Baltasar et al. ${ }^{8}$ reported the concentration-pressure phase diagram of sodium dodecanoate obtained from speedof-sound measurements, where the formation of a gel-like 
phase seen as lamellae of surfactants was observed. This transition was also perceived by our group using Molecular Dynamics (MD) simulations in an aqueous system with SDS micelles. $^{7}$

Unfortunately, there is not yet a detailed description that pressure can exert on nonionic surfactants as there is for the ionic ones. In this line, we can mention Hara's group work where they found that the aggregation number of the Triton X100 (TRX100) micelle reached its minimum around $150 \mathrm{MPa}$ and then it increased to almost 230 when the pressure varied from $200 \mathrm{MPa}$ to $500 \mathrm{MPa} .{ }^{6}$ For this system, the maximum CMC was obtained at pressures near 100-110 MPa.

It is noteworthy that the measured value is almost identical to the pressure at which the SDS micelle ${ }^{21}$ also attains the maximum CMC. Nonetheless, the solvation for TRX100 decreases, opposite to what happens to SDS, suggesting a different hydration structure surrounding micelles. ${ }^{1}$

Currently, there is a deficiency in the literature on the effect of high pressure on non-ionic surfactants at the molecular level. At present, few models of Triton X-100 are reported in the literature ${ }^{22-25}$ and only two articles can be found concerning the self-assembly of Triton X-100 micelles as a function of temperature and surfactant concentration using computational methodologies. These studies were carried out using all-atom MD simulations ${ }^{23}$ and coarse-grained (CG) model with reverse mapping, ${ }^{22}$ obtaining an atomistic model of the micellar clusters from mesoscale simulations.

In the present work, we have studied the self-assembly of micelles composed of the non-ionic surfactant TRX100 at the atomic scale and the effect of hydrostatic pressures on a self-assembled micelle. We have obtained detailed and valuable information about the aqueous solution conformation of TRX100 micelles (size and shape during the self-assembly process) and have monitored changes in the solvation dynamics and observed the consequent molecular confinement as a consequence of increasing pressures.

\section{COMPUTATIONAL METHODS}

The TRX100 molecule is composed of two portions: a hydrophobic region formed of an atomic set consisting of an octyl phenyl group $\left(\mathrm{CR} 1-\mathrm{C}-\mathrm{CH}_{n}\right)$ and a hydrophilic one encompassing a sequence of ether bonds $\mathrm{CH} n-\mathrm{OA}$ (polyoxyethylene group). Notwithstanding, the TRX100 molecule does not present a well-marked hydrophilic-hydrophobic boundary.,26 In fact, 2D nuclear overhauser effect spectroscopy (NOESY) data indicated intermolecular interactions by correlating resonance signals between the methyl groups located at the extremities, along with the aromatic protons belonging to the hydrophobic region, and the first three methylene groups from the polyoxyethylene chain during micellization. ${ }^{26}$ Therefore, we defined these three particular methyl groups as being hydrophobic (Fig. 1).

Subsequently, we built a molecular topology for TRX100 based on Molecular Mechanics (MM) calculations using a GROMOS 53A6 $O X Y+D$ force field, which was calibrated and optimized to describe ether and polyether chemical groups in aqueous solution. ${ }^{27}$ The molecular geometry of a single TRX100 molecule was optimized using ab initio methods

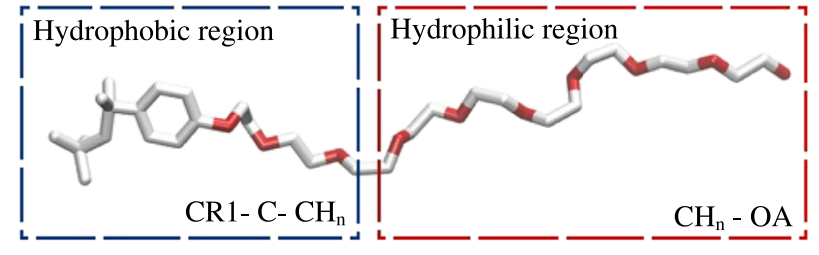

FIG. 1. The Triton X-100 molecule employed in our MD simulations was divided into two regions: hydrophilic and hydrophobic. Red and blue boxes indicate hydrophilic and hydrophobic regions, respectively. Atom types were defined according to the Gromacs library. Red atoms indicate oxygen atoms.

with the B3LYP/6-311 $+\mathrm{G}(\mathrm{d})^{28}$ level of theory using the Gaussian03 $3^{29}$ program. Using this setting, Denkova and coworkers ${ }^{26}$ studied the mutual spatial arrangement of surfactant molecules in the aggregated state regarding the micelle shape. Their $a b$ initio calculations were validated using Nuclear Magnetic Resonance (NMR) spectroscopy.

Topology for TRX100 with 9.5 oxyethylene units is shown in Fig. 1, while molecular parameters compatible with the $53 \mathrm{~A} 6_{O X Y+D}$ force field are shown in Table I. Selection and assignment of dihedral angle parameters were based on the work of Fuchs et al., ${ }^{27}$ wherein they compared potential energy profiles corresponding to rotations around the dihedral angles $\mathrm{O}-\mathrm{C}-\mathrm{C}-\mathrm{O}$ and $\mathrm{C}-\mathrm{C}-\mathrm{O}-\mathrm{C}$ for neighboring diethers and polyethers. Parameterization was accomplished by fitting the conformational transitions corresponding to a gradual population shift from trans to gauche of the $\mathrm{O}-\mathrm{C}-$ $\mathrm{C}-\mathrm{O}$ and $\mathrm{C}-\mathrm{C}-\mathrm{O}-\mathrm{C}$ dihedral angles, in agreement with the experimental data about hydration properties of such systems (Table I).

The group charges for the hydrophilic and hydrophobic regions in the TRX100 molecule were defined according to NMR data, using Nuclear Overhauser Effect Spectroscopy (NOESY) and diffusion-ordered NMR Spectroscopy $(\mathrm{DOSY})^{26}$ techniques, i.e., the first $\mathrm{O}-\mathrm{C}-\mathrm{C}-\mathrm{O}-\mathrm{C}$ group from the polyoxyethylene chain (hydrophilic region) is part of the hydrophobic region along with the octyl phenyl group (Fig. 1). The values of atomic partial charges for each group were adopted from the $53 \mathrm{~A} 6_{O X Y+D}$ force field ${ }^{27}$ (Table I). For more details, see the molecular topology of TRX100 in the supplementary material.

\section{A. Simulation parameters}

MD simulations were carried out using the GROMACS 4.6.3 package. $^{30,31}$ The GROMOS $53 \mathrm{~A} 6_{O X Y+D}{ }^{27}$ force field was included in the Gromacs library. Water molecules were modeled using the simple point charge extended, SPC/E, model. ${ }^{32}$ Simulations began from a random spatial distribution of the TRX100-water mixture according to the experimental value of the aggregation number $\left(\mathrm{N}_{A g g}=143-287\right){ }^{3-5,26}$

Thus, we hydrated a cubic box $(\mathrm{X}=\mathrm{Y}=\mathrm{Z}=11.826 \mathrm{~nm})$ containing a homogeneous mixture composed of 200 TRX100 molecules and 49034 water molecules, resulting in a concentration of $0.2 \mathrm{~mol} / \mathrm{l}$. We performed a first energy minimization stage utilizing the steepest descent method during 5000 steps, followed by a second one using the conjugate gradient algorithm until the energy gradient of $\leq 10 \mathrm{~kJ} \mathrm{~mol}^{-1} \mathrm{~nm}^{-1}$ was achieved. 
TABLE I. Molecular parameters of TRX100 according to the GROMOS $53 \mathrm{~A} 6_{O X Y+D}$ force field. ${ }^{27}$

\begin{tabular}{|c|c|c|c|}
\hline Covalent bonds ${ }^{\mathrm{a}}$ & $\mathrm{b}_{0}(\mathrm{~nm})$ & \multicolumn{2}{|c|}{$\mathrm{k}_{b}\left(10^{6} \mathrm{~kJ} \mathrm{~mol}^{-1} \mathrm{~nm}^{-4}\right)$} \\
\hline CR1-CR1 & 0.139 & \multicolumn{2}{|c|}{10.8} \\
\hline $\mathrm{CH}_{n}-\mathrm{CH}_{n}$ & 0.153 & \multicolumn{2}{|c|}{7.15} \\
\hline $\mathrm{CH}_{n}-\mathrm{C}$ & 0.153 & \multicolumn{2}{|c|}{7.15} \\
\hline CR1-OA & 0.136 & \multicolumn{2}{|c|}{10.2} \\
\hline $\mathrm{CH}_{n}-\mathrm{OA}$ & 0.143 & \multicolumn{2}{|c|}{8.18} \\
\hline Bond angles ${ }^{\mathrm{b}}$ & Angles $\theta_{0}$ & \multicolumn{2}{|c|}{$\mathrm{k}_{\theta}\left(\mathrm{kJ} \mathrm{mol}^{-1}\right)$} \\
\hline $\mathrm{C}-\mathrm{CH}_{n}-\mathrm{C}$ & 109.5 & \multicolumn{2}{|c|}{520.0} \\
\hline $\mathrm{CH}_{n}-\mathrm{C}-\mathrm{CH}_{n}$ & 109.5 & \multicolumn{2}{|c|}{520.0} \\
\hline $\mathrm{CH}_{n}-\mathrm{C}-\mathrm{CR} 1$ & 109.5 & \multicolumn{2}{|c|}{520.0} \\
\hline $\mathrm{C}-\mathrm{CR} 1-\mathrm{CR} 1$ & 120.0 & \multicolumn{2}{|c|}{560.0} \\
\hline CR1-CR1-CR1 & 120.0 & \multicolumn{2}{|c|}{560.0} \\
\hline CR1-CR1-OA & 120.0 & \multicolumn{2}{|c|}{560.0} \\
\hline $\mathrm{CR} 1-\mathrm{OA}-\mathrm{CH}_{n}$ & 111.0 & \multicolumn{2}{|c|}{530.0} \\
\hline $\mathrm{OA}-\mathrm{CH}_{n}-\mathrm{CH}_{n}$ & 111.0 & \multicolumn{2}{|c|}{530.0} \\
\hline $\mathrm{CH}_{n}-\mathrm{OA}-\mathrm{CH}_{n}$ & 111.0 & \multicolumn{2}{|c|}{530.0} \\
\hline Dihedral angles ${ }^{\mathrm{c}}$ & $\mathrm{k}_{\phi}\left(\mathrm{kJ} \mathrm{mol}^{-1}\right)$ & $\cos \delta_{\phi}$ & $\mathrm{m}_{\phi}$ \\
\hline $\mathrm{C}-\mathrm{CH}_{n}-\mathrm{C}-\mathrm{CH}_{n}$ & 5.44 & 1 & 3 \\
\hline $\mathrm{CH}_{n}-\mathrm{C}-\mathrm{CH}_{n}-\mathrm{C}$ & 5.44 & 1 & 3 \\
\hline $\mathrm{CH}_{n}-\mathrm{C}-\mathrm{CR} 1-\mathrm{CR} 1$ & 41.8 & -1 & 2 \\
\hline $\mathrm{CR} 1-\mathrm{CR} 1-\mathrm{OA}-\mathrm{CH}_{n}$ & 7.11 & -1 & 2 \\
\hline $\mathrm{CH}_{n}-\mathrm{CH}_{n}-\mathrm{OA}-\mathrm{CH}_{n}$ & 0.931 & -1 & $1^{\mathrm{d}}$ \\
\hline $\mathrm{OA}-\mathrm{CH}_{n}-\mathrm{CH}_{n}-\mathrm{OA}$ & 6.787 & 1 & $3^{e}$ \\
\hline $\mathrm{OA}-\mathrm{CH}_{n}-\mathrm{CH}_{n}-\mathrm{OA}$ & 6.787 & 1 & $3^{e}$ \\
\hline $\mathrm{OA}-\mathrm{CH}_{n}-\mathrm{CH}_{n}-\mathrm{OA}$ & 6.787 & -1 & $1^{\mathrm{e}}$ \\
\hline
\end{tabular}

Atomic partial charges q(e)

Hydrophobic region

\begin{tabular}{llc} 
& $\mathrm{CR} 1$ & 0.000 \\
& $\mathrm{C}$ & 0.000 \\
& $\mathrm{CH}_{n}$ & 0.000 \\
& $\mathrm{OA}$ & 0.000 \\
Hydrophilic region & & \\
Ether & $\mathrm{CH}_{n}$ & 0.290 \\
& $\mathrm{OA}$ & -0.580 \\
Alcohol & $\mathrm{OA}$ & -0.290 \\
\hline
\end{tabular}

a Bond length $b_{0}$ and force constant $k_{b}$ for bond stretching.

${ }^{\mathrm{b}}$ Bond angle $\theta_{0}$ and force constant $k_{\theta}$ for bond angle bending.

${ }^{\mathrm{c}}$ Multiplicity $\mathrm{m}_{\phi}$, phase-shift $\operatorname{cosine} \cos \delta_{\phi}$, and force constant $k_{\phi}$.

${ }^{\mathrm{d}}$ The parameters for the dihedral angles $\mathrm{C}-\mathrm{C}-\mathrm{O}-\mathrm{C}$ were selected for a single trans conformation.

${ }^{\mathrm{e}}$ The parameters for the dihedral angles $\mathrm{O}-\mathrm{C}-\mathrm{C}-\mathrm{O}$ have been chosen in three successive conformers, gauche-gauche-trans.

Afterward, the system was simulated in the NpT ensemble at $\mathrm{p}=1 \mathrm{~atm}$ and $\mathrm{T}=300 \mathrm{~K}$ using the $\mathrm{V}$-rescale thermostat ${ }^{33}$ (coupling constant $\tau=0.1 \mathrm{ps}$ ) and the Berendsen barostat ${ }^{34}$ (coupling constant $\tau=1.0 \mathrm{ps}$ ) for temperature and pressure control, respectively. During simulations, TRX100 bonds were constrained using the LINCS algorithm, ${ }^{35}$ while electrostatic interactions were computed using the particle mesh Ewald method. ${ }^{36}$ A cutoff of $1.0 \mathrm{~nm}$ was applied for the van der Waals and Coulomb interactions. The integration time step was $2 \mathrm{fs}$ throughout the simulations.

Before the high-pressure regime production phase, a 200 ns equilibration stage was performed without using any restraints to produce a self-assembled micelle. The criterion adopted to define the micellization was based on a geometrical classification where the cluster size distribution must be within a specified cutoff distance. During the 200 ns of simulation, all monomers converge to one micelle.

\section{B. Pressure effects}

Once the micelle was self-assembled, this configuration was taken as the starting point for the simulations under pressure. Thus, the micellar system was coupled to a hydrostatic pressure bath, and the pressure was increased at regular increments of 200 bars each until a final pressure of 4000 bars was achieved. Additions occurred systematically every $20 \mathrm{~ns}$. An equilibration run of $15 \mathrm{~ns}$ and a collection period of $5 \mathrm{~ns}$ were performed for each pressure stage.

The kinetic/dynamic processes can be considerably slowed down with pressure. ${ }^{37}$ Even in self-assembled micellar systems directly coupled to high pressures, it is almost impossible to see conformational transitions. By contrast, when the systems are coupled to gradual increases in pressure, they do not lose mobility immediately and give rise to new configurations. ${ }^{7}$ The $20 \mathrm{~ns}$ of trajectory in each pressure increase was selected following criteria determined in previous studies performed by our group. ${ }^{7,38}$ Basically, the simulation time was determined on the ground of the stability of the potential energy for each pressure increase. Also, we ensured that the pressure coupling manages to represent the liquidliquid transition from a low-density to high-density state in water.

Finally, trajectories in the phase space were saved every 20 ps to analyze structural features. All calculations were carried out on a Linux server Intel Xeon E5-2620 $2 \mathrm{GHz}$ Six Core Processor with NVIDIA GeForce GTX 780 Ti. Simulation data were analyzed using the GROMACS tool package.

\section{RESULTS AND DISCUSSION}

\section{A. Self-assembly and micellization}

MD simulations of TRX100 in the aqueous solution provided new insights into the self-assembly and micellization of the surfactant at a concentration above the CMC, $\sim 200 \mathrm{mM} .{ }^{6,21,39,40}$ As a first validation of the TRX100 computational model, we evaluated the kinetics of self-assembly and the spontaneous formation of micellar structures. To this end, we computed the hydrophilic, hydrophobic, and total values of the solvent-accessible surface area (SASA) of TRX100 over $200 \mathrm{~ns}$. Micelles fully formed at around the first $20 \mathrm{~ns}$, although aggregation kinetics started around the first ten ns. This process seemed to be driven by the gathering of the nonpolar region of TRX100 molecules creating the hydrophobic core (Fig. 2). On the other hand, the interaction of polyoxyethylene chains with water molecules with the resultant structuration of their hydration shell took longer. It was observed that the polyoxyethylene chains were exposed to the solvent and demanded more time (above $20 \mathrm{~ns}$ ) to compact and stabilize the micellar system. Full micellization was reached at nearly $80 \mathrm{~ns}$, with the consequent stabilization of the SASA value at $405 \pm 9.1 \mathrm{~nm}^{2}$. 


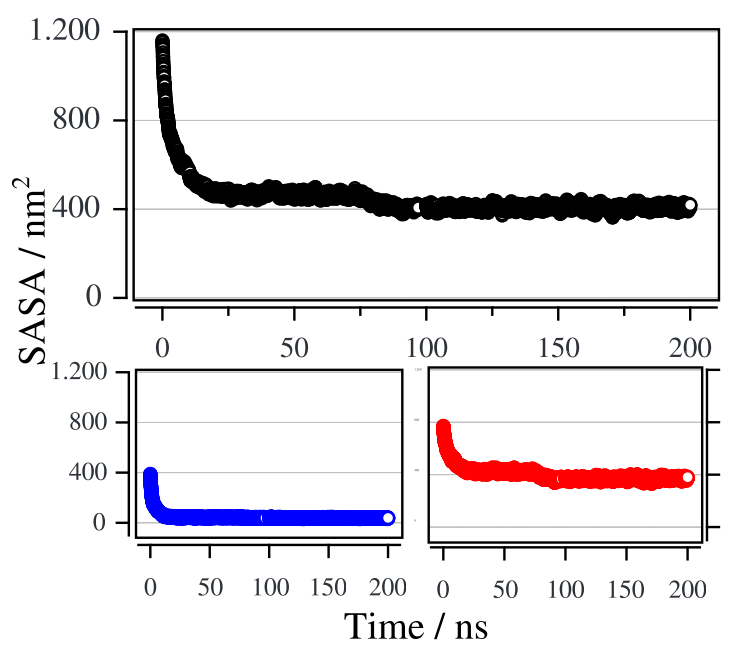

FIG. 2. Area of TRX100 exposed to the solvent during the micelle self-assembly. (Top, black line) Total SASA. (Bottom) Hydrophobic and hydrophilic SASA (blue and red lines, respectively).

Also, the analysis of the aggregation number $\left(\mathrm{N}_{A g g}\right)$ and the enumeration of the number of clusters $\left(\mathrm{N}_{\text {Clust }}\right)$ during simulation time contributed to unveil the micellar self-assembly. We utilized the nonhierarchical cluster algorithm GROMOS ${ }^{41}$ to identify the TRX100 aggregates. A cluster was regarded as such when all the monomers were within a specified cutoff $\left(1.0 \mathrm{~nm}^{22}\right)$. So, the TRX100 molecule that maximized the number of neighbors was taken as the center of the cluster and formed, together with all its neighbors, a first group.

Upon $80 \mathrm{~ns}$ of simulation, a single micelle was established achieving an $\mathrm{N}_{A g g}=200$ [Fig. 3(a) (maximum $\mathrm{N}_{A g g}$ ) and 3(b) (average $\mathrm{N}_{A g g}$ )]. As expected, the self-aggregation process, computed by monitoring of the aggregation number, correlates (coefficient of determination $\mathrm{r}=0.93, \mathrm{p} \leq 0.001$ ) well with the diminishing of the area of TRX100 molecules exposed to the solvent during the micelle self-assembly [Fig. 3(c) and Fig. 1(S) in the supplementary material]. The $\mathrm{N}_{\text {Clust }}$ value decreased rapidly during the first $20 \mathrm{~ns}$ and formed an aggregate with a maximum $\mathrm{N}_{A g g}$ of $\sim 150$ monomers
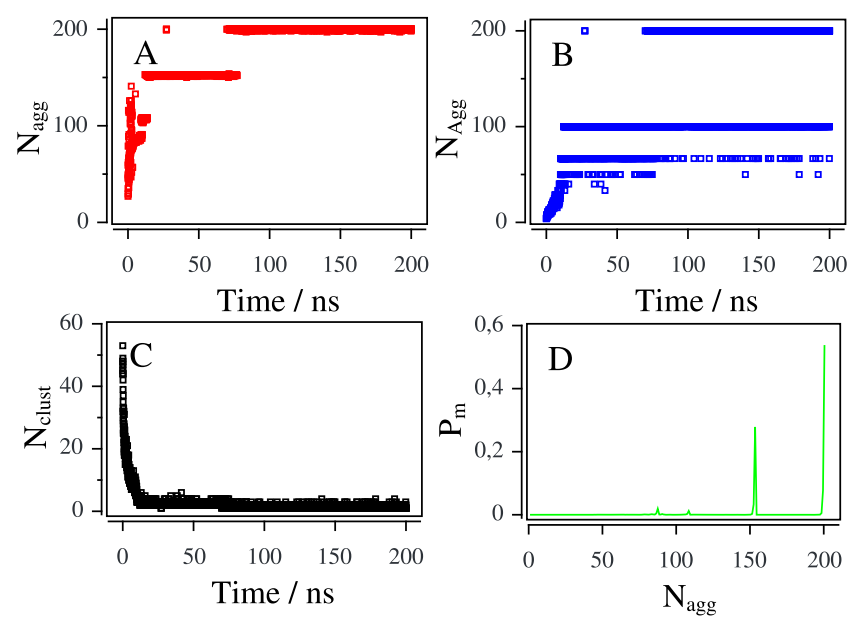

FIG. 3. [(a) and (b)] Maximum and average aggregation number of TRX100 molecules over the simulation time, respectively. (c) The number of clusters formed. (d) The most likely aggregation number.
[Fig. 3(a)]. From Fig. 3(c), a relaxation time of 3.97 ns was estimated.

Finally, the probability $P_{m}$ of finding a cluster of $m$ monomers can be defined as

$$
P_{m}=\frac{n(m) m}{N},
$$

where $n(m)$ is the number of aggregates of $m$ monomers and $N$ the total monomers. Then, $n(m) / N$ is considered as a relative frequency and $P_{m}$ can satisfy the normalization condition

$$
\sum_{t=1}^{N} P_{m}=1 .
$$

Thus, the most likely aggregation number of micelles during the total simulation time corresponded to 200 monomers [Fig. 3(d)].

Moreover, we remark that during the simulation time analyzed in this work, all monomers in the simulation box formed a single micellar assembly. We are aware that bigger molecular systems (>200) might form micelles with higher aggregation numbers. However, we emphasize that our primary goal was to observe the formation of a micelle by self-assembly with an aggregation number in agreement with the available experimental values in order to analyze the molecular confinement and changes in its solvation dynamics as a consequence of increasing pressures. Also, we recognize that concentration effects can influence the size and shape of micellar aggregates, where shape transitions in isotropic micellar phase are clearly evident. ${ }^{22}$ However, this issue is out of the purpose of this work, and more simulations will be necessary to show the micellar geometric evolution as a function of the concentration. We hope to address this important aspect in future work.

To fully characterize assembly, we also analyzed some structural parameters. The micelle shape was estimated using the eccentricity parameter, $\varepsilon$, defined as

$$
\varepsilon=1-\frac{I_{\min }}{I_{\text {avg }}},
$$

where $I_{\min }$ is the moment of inertia with the smallest magnitude along the principal axes and $I_{\text {avg }}$ is the average of the three moments of inertia. $\varepsilon$ can adopt values in the range 0 to 1 . Thus, aggregates with $\varepsilon=0$ correspond to a highly symmetrical shape with spherical geometry, while values different from zero indicate drifts to a more elliptical shape. . $3,42,43$

By monitoring the variation of $\varepsilon$ over the simulation time, we conclude that the aggregate cannot be considered entirely spherical but resembling a spheroid $(\varepsilon=0.15-0.3)$. $\varepsilon$ values correlate well with the variations of the radius of gyration $(\mathrm{Rg})$ on the principal axes of the micelle [Fig. 2(S) in the supplementary material]. The variations observed in the eccentricity, moment of inertia, and Rg [see Figs. 4(a) and 4(b) and Fig. 2(S) in the supplementary material, respectively] during the first $80 \mathrm{~ns}$ of trajectory can be ascribed to polydispersity of micellar assemblies [see Fig. 3(S) in the supplementary material]. Therefore, the highest value observed in Fig. 4 and Fig. 2(S) of the supplementary material around $50 \mathrm{~ns}$ refers to the coexistence of two micellar aggregates which eventually converge to a unique micellar structure [Fig. 3(S) in the supplementary material]. 

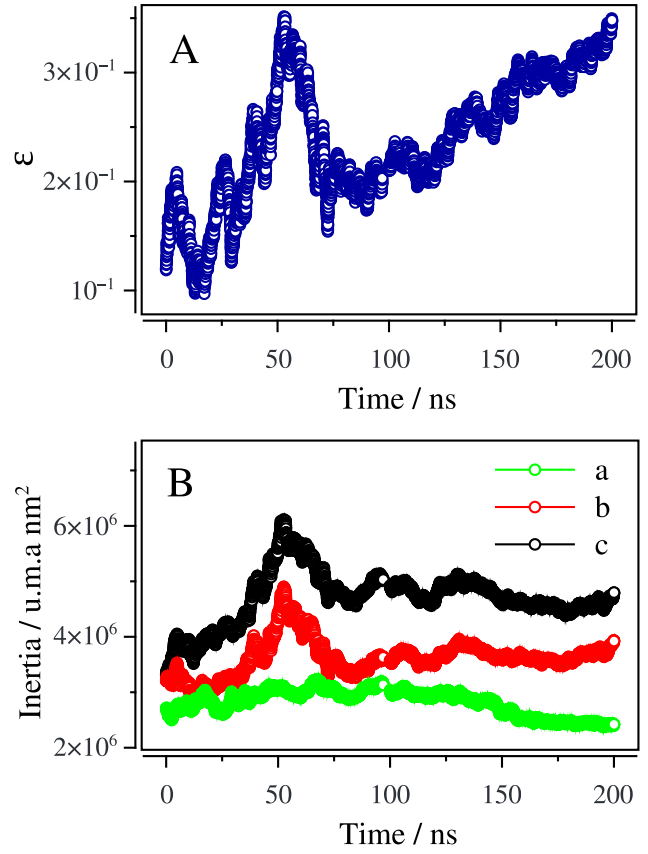

FIG. 4. Structural parameters computed throughout the trajectory. (a) Eccentricity $\varepsilon$. (b) Moment of inertia along the principal axes.

From $\mathrm{Rg}$ values, we estimated the main inertia axes of the micelle (semi-axes $a, b$, and $c$ ) and were able to establish a relationship between these. Thus, when $c<a$, the aggregate adopts a spheroid oblate shape, and when $c>a$, a prolate spheroid is formed. In the particular case in which $a$ and $c$ semi-axes are equal, the micelle assumes a spherical form.

Figure 4(b) shows that once the micelle is self-assembled ( $\sim 80 \mathrm{~ns})$ a prolate spheroid is apparent. This result agrees with the ones obtained by De Nicola et al.,22 who showed, by performing a theoretical analysis with a hybrid scheme MD-CG (Molecular Dynamics and Coarse-Grained) micelles of TRX100, that $\mathrm{N}_{A g g}>140$ characterizes prolate ellipsoidal shapes.

Alongside, NOESY NMR data obtained by Denkova et $a l .{ }^{26}$ confirmed the same eccentricity trend, where Triton X100 micelles were found to be almost spherical at low surfactant concentrations, reaching ellipsoidal shapes at higher levels of the surfactant.

\section{B. Pressure effects}

To study the effect of pressure exerted on this kind of molecular assemblies, a binary system, composed of a TRX100-micelle in aqueous solution, was submitted to the action of increasing pressure. At 1 bar (atmospheric pressure), micelles presented precise polar heads, consisting of ether groups, with their hydrophobic tails oriented toward the inner part of the molecular structure, forming a prolate spheroid micelle [Fig. 4(S) in the supplementary material]. When the micelle was subjected to higher pressures (200 bars up to 4000 bars), its eccentricity dropped below 0.1 at 200 bars of pressure, indicating a more spherical arrangement of the micelle as a consequence of the increasing pressure [Fig. 5(a)].

By assuming that the micelle adopts a spherical shape under high pressures, the $\mathrm{Rg}$ can be calculated as the
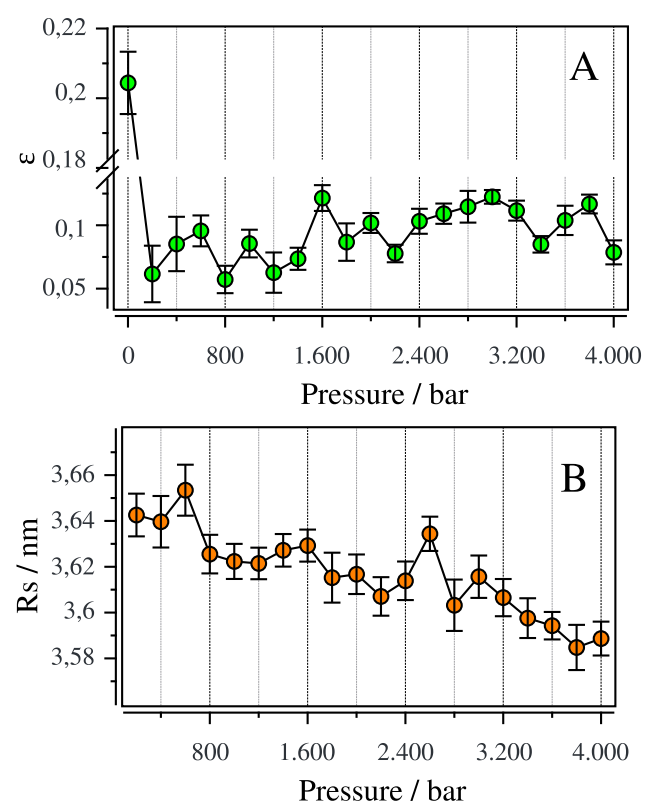

FIG. 5. Pressure effects in the micellar geometry. (a) Eccentricity, $\varepsilon$ (1-4000 bars); (b) the radius of the micelle, Rs (200-4000 bars). Dots and error bars represent the averages and the standard deviations, respectively.

root-mean-square distance between the center of mass of the micelle and its ends [Fig. 5(S) in the supplementary material]. Thus, the radius of the micelle, Rs, can be calculated from the relation to $\mathrm{Rg}$ according to ${ }^{23,43}$

$$
R s=\sqrt{\frac{5}{3}} R g .
$$

Concomitantly, we observed a gradual decrease of the Rs value, from $3.64 \mathrm{~nm}$ (200 bars) to $3.58 \mathrm{~nm}$ (4000 bars), indicating a reduction of $1.48 \%$ in Rs under the high-pressure regime.

Hara et al. ${ }^{21}$ carried out a study in which they measured volume changes induced by the increasing pressure. They observed that the compressibility was higher for micellar systems than for those in which TRX100 molecules were diluted in water. Coincidentally, our simulations indicated a variation in the compressibility of TRX100 micelles of $57.6 \%$ with increasing pressure [Fig. 6(S) in the supplementary material].

Despite fluorescence data ${ }^{21}$ showed a linear reduction in the micellar volume with increasing pressure, from $+4.6 \mathrm{~cm}^{3}$ $\mathrm{mol}^{-1}$ at atmospheric pressure to $-7.0 \mathrm{~cm}^{3} \mathrm{~mol}^{-1}$ at 3000 bars, it is likely that the CMC of TRX100 increases with growing pressure, leading TRX100 molecules into the aqueous phase. Strikingly, SASA variations due to the high-pressure effects result in increasing values of Rs (see Sec. III B and Fig. 6) suggesting a more significant exposure of the TRX100 molecules to the solvent.

\section{Solvent effects}

Our results suggest that the fluctuations in the Rs are mainly due to the increase in the solvation of the hydrophilic area. Although the total SASA decreased in the micelle due to the increase in pressure, the hydrophilic component increased, which is probably due to a rearrangement of the monomer headpieces. 

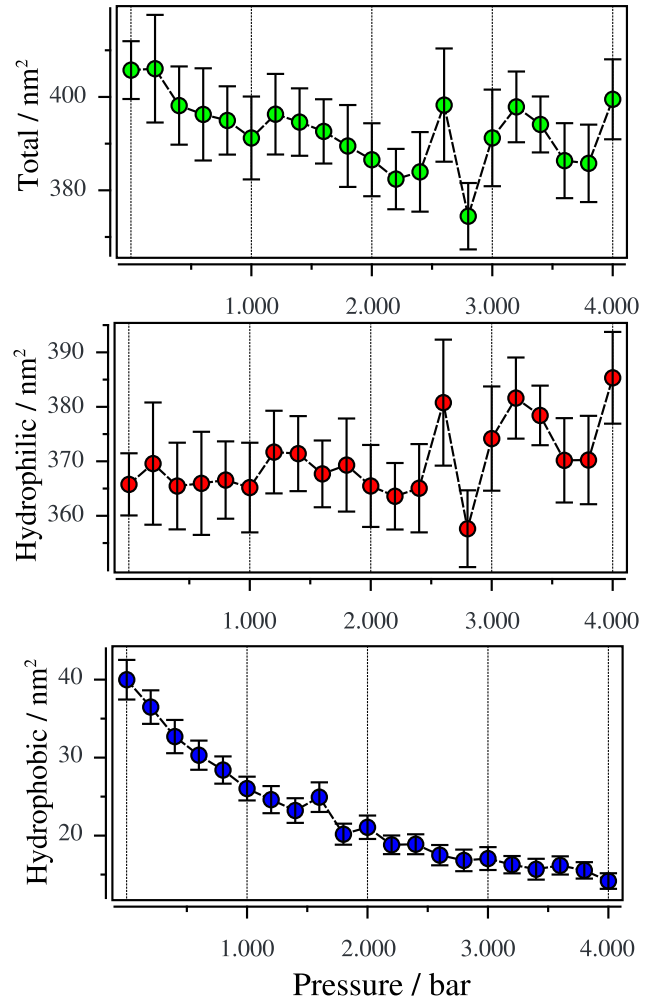

FIG. 6. Pressure effects in the solvent-accessible surface area, SASA, total (green dots), hydrophilic (red dots), and hydrophobic (blue dots). Dots and error bars indicate the averages and the standard deviations, respectively.

Concomitantly, the hydrophobic core, whose SASA decreased with pressure, prevents further contact with water molecules (Fig. 6). Comparative studies on the solvation dynamics among different micellar environments [neutral micelle, TRX100 and ionic micelle, and sodium dodecyl sulfate (SDS)] as a function of pressure ${ }^{1}$ showed that a broader exhibition of the polar headpieces to a larger area facilitated the formation of hydrogen bonds with water. The exhibition also resulted in the increment of the micellar aggregation number. As a consequence, TRX100 molecules generated a thicker and a better-organized hydration shell than SDS micelles did under similar conditions. ${ }^{1,44}$

In our simulations, we observed an increment of the number of water-TRX100 hydrogen bonds with increasing pressure, resulting in the restructuring of water molecules around the micelle producing a change in the micellar environment [Fig. 7(S) in the supplementary material].

In this regard, we analyzed the solvation dynamics calculating the density of water molecules in the vicinity of the hydrophilic region using the radial distribution function, $\mathrm{g}(\mathrm{r})$. As pressure increases, the first and second peaks of the $\mathrm{g}(\mathrm{r})$ function get higher due to the compression that water molecules exert around the hydrophilic region of the TRX100 micelle (Fig. 7). As long as the position of the first peak remains unaltered, the localization of the second peak is displaced to the left, indicating a more condensed arrangement of the second hydration layer. Interestingly, although the high pressures compressed the micelle reducing consequently the hydrophobic core (Fig. 6), the rise in total and hydrophilic SASA values can be due to the entrance of water into the

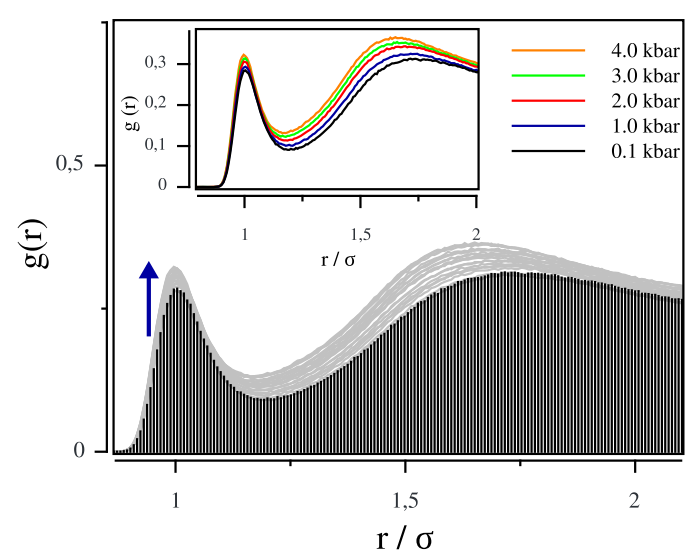

FIG. 7. Radial distribution function, g(r), between oxygen atoms of water molecules around the hydrophilic region (oxygen atoms in the polyether) in the TRX100 micelle at high pressures. Arrows indicate the direction of the increased pressure. The inset shows density changes every $1.0 \mathrm{kbar}$. The normalization value refers to the diameter of a water molecule $(0.275 \mathrm{~nm})$.

micelle [see Fig. 8 and Figs. 8(S) and 9(S) in the supplementary material].

Thus, changes in the hydration shell around the hydrophilic region could be responsible for modifications in the structural dynamics of the micelle producing fluctuations of Rs values. Small-angle neutron scattering (SANS) studies performed on $\mathrm{C}_{i} \mathrm{E}_{j}$ (n-alkyl polyoxyethylene ether) micelles showed that the compression of the surfactant produces changes in the partial molecular volume inducing the dehydration of the polar heads concomitant with the hydrophilic micelle shell collapse. ${ }^{45}$

On the other hand, Hara and co-workers ${ }^{21}$ suggest that the primary effect of high pressures on variations of CMC is due to water penetration into the micelles and changes in the partial molar volume. According to our simulations, one of the effects increasing pressure produces can be the migration of water molecules toward the hydrophobic micellar core (Fig. 8).

It is well known that micellar systems composed of TRX100 molecules experience a turnover behavior in the aggregation number $\mathrm{N}_{A g g}$, which coincides with $\mathrm{CMC}$ variations when the pressure increases. ${ }^{6,21}$ Due to the time scale

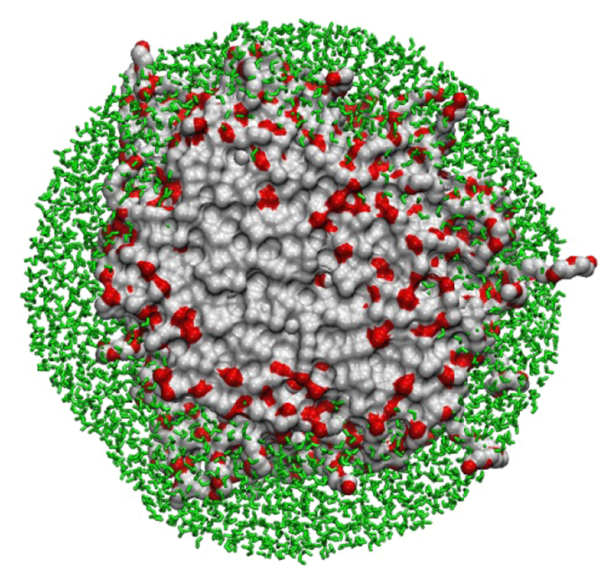

FIG. 8. Snapshot of the TRX100 micelle to $4.0 \mathrm{kbar}$. In this image, we can see a cross section of the micelle (oxygen and carbon atoms in red and white, respectively) and water molecules around (in green). 
involved in our simulations, this behavior was not observed because the dynamics are slowed down with pressure. Our results show, in agreement with the experimental data, ${ }^{1,44}$ an increase in the number of the hydrogen bonds between headgroups and water molecules [Fig. 7(S) in the supplementary material]. So, water molecules are more likely to invade the polar headgroups under the pressure regime (Figs. 6 and 8). Conversely, the application of pressure weakens the strength of the hydrogen bonds between water molecules, restructuring the hydrogen bond network [Fig. 10(S) in the supplementary material]. Thus, under the pressure regime above 2200 bars, the liquid water shifts from a low-density (LD) into a highdensity (HD) state, ${ }^{46-48}$ favoring the upsurge in the solvation of the hydrophilic area.

Finally, we calculated the volume change in the TRX100 micelle under pressure using the computational tool trjVoronoi. ${ }^{49,50}$ We observed that the volume of the micelle decreases following Le Chatelier's principle [Fig. 11(S) in the supplementary material]. However, under the high-pressure regime, above 2200 bars, water molecules invade the micelle (Figs. 7 and 8) increasing the solvation of the hydrophilic area (Fig. 6). Because most peculiar properties of water are due to the existence of a hydrogen bond network, ${ }^{14}$ the changes at high pressures can be understood as evidence of a shift in the water structure [Fig. 10(S) in the supplementary material], moving from an open tetrahedral structure (LD) to a compact hexagonal one (HD) ${ }^{48}$ It is worth mentioning that the only water model representing the liquid-liquid transition (LD to $\mathrm{HD})$ at the pressure experimentally reported $(\sim 0.2 \mathrm{GPa})^{48}$ is the SPC/E model so far.

Hence, our results suggest that changes in the hydration shell around micelles could be mainly due to the pressure effect on the hydrogen bonding network with water. In particular, water penetration into the micelle is likely related to the occurrence of a high-density state of water above 2200 bars.

\section{CONCLUSIONS}

In this work, a molecular parameterization of Triton $\mathrm{X}-100$ molecules was created, optimized, and validated. The computational molecular model was capable of selfassembling and forming a non-ionic micelle. New geometric and aggregation parameters were also in agreement with current experimental data. $3,6,26$

We have observed the self-assembly of a prolate ellipsoid micelle, starting from an initial configuration of randomly placed free monomers. Later, the micelle was coupled to a high-pressure barostat, which produced a geometric transition of the micelle from an ellipsoid to a sphere. At the same time, the micellar radius slightly increased, mainly due to changes in the solvation dynamics. As it is well known, the water structure is highly influenced by pressure, generating structural anomalies in the liquid water. ${ }^{46,51}$ Therefore, the first coordination shell around the solute is destabilized, the hydrogen bonds are redistributed, and the water density is modified.$^{46}$ Herein, water acts as an unstructured liquid, ${ }^{47}$ capable of penetrating into the micelle due to both the restructuring of hydrogen bond network and density changes.
Although the simulation time was not sufficient to observe exhibition of the hydrophobic core to the solvent, changes in the radius of the micelle were closely related to the entrance of water into the hydrophilic region, reinforcing the idea that the primary effect of high pressures on water penetration. Furthermore, migration of water molecules from the bulk to the micelle can also be directly involved with the variation of CMC, aggregation number, and partial molar volume..$^{6,21}$

Finally, we were not able to observe changes in the aggregation number due to pressure effects in our simulations. However, it is likely that experiments carried out with more diluted systems may evidence them. Still, the aggregation dynamics, geometry, and solvation properties have been well represented by our model.

\section{SUPPLEMENTARY MATERIAL}

See supplementary material for additional information concerning linear regression and radius of gyration analysis during micellization, micellar assemblies and micellar geometry, pressure effects in radius of gyration, isothermal compressibility, hydrogen bond distribution in water-TRX100 and water-water, water penetration into the micelles, volume changes, and the molecular topology of the TRX100 molecule.

\section{ACKNOWLEDGMENTS}

The authors would like to thank Dr. Manuel Carlevaro for his valuable comments. This work was funded by the National Research Council of Argentina (CONICET) and Carlos Chagas Filho Foundation for Research Support of the Rio de Janeiro State (FAPERJ). YR. Espinosa was supported by the CONICET and FAPERJ.

${ }^{1}$ K. Hara, N. Baden, and O. Kajimoto, J. Phys.: Condens. Matter 16, S1207 (2004).

${ }^{2}$ S. Paula, W. Sues, J. Tuchtenhagen, and A. Blume, J. Phys. Chem. 99, 11742 (1995).

${ }^{3}$ R. J. Robson and E. A. Dennis, J. Phys. Chem. 81, 1075 (1977).

${ }^{4}$ V. Patel, D. Ray, V. K. Aswal, and P. Bahadur, Colloids Surf., A 450, 106 (2014).

5 J. Molina-Bolivar, J. Aguiar, and C. C. Ruiz, J. Phys. Chem. B 106, 870 (2002).

${ }^{6}$ N. Baden, O. Kajimoto, and K. Hara, J. Phys. Chem. B 106, 8621 (2002).

${ }^{7}$ Y. R. Espinosa and J. R. Grigera, RSC Adv. 5, 70005 (2015).

${ }^{8}$ E. H. Baltasar, M. Taravillo, P. D. Sanz, V. G. Baonza, and B. Guignon, Langmuir 30, 7343 (2014).

${ }^{9}$ J.-B. Rouget, T. Aksel, J. Roche, J.-L. Saldana, A. E. Garcia, D. Barrick, and C. A. Royer, J. Am. Chem. Soc. 133, 6020 (2011).

${ }^{10}$ C. A. Royer, Biochim. Biophys. Acta 1595, 201 (2002).

${ }^{11}$ C. L. Dias, Phys. Rev. Lett. 109, 048104 (2012).

${ }^{12}$ J. Roche, J. A. Caro, D. R. Norberto, P. Barthe, C. Roumestand, J. L. Schlessman, A. E. Garcia, and C. A. Royer, Proc. Natl. Acad. Sci. U. S. A. 109, 6945 (2012).

${ }^{13}$ B. Pereira, S. Jain, S. Sarupria, L. Yang, and S. Garde, Mol. Phys. 105, 189 (2007)

${ }_{14}^{14}$ J. R. Grigera and A. N. McCarthy, Biophys. J. 98, 1626 (2010).

${ }^{15}$ D. Bossev, S. Kline, J. Israelachvili, and M. Paulaitis, Langmuir 17, 7728 (2001).

${ }^{16}$ S. Hamann, J. Phys. Chem. 66, 1359 (1962).

${ }^{17}$ S. Kaneshina, M. Tanaka, T. Tomida, and R. Matuura, J. Colloid Interface Sci. 48, 450 (1974).

${ }^{18}$ N. Nishikido, M. Shinozaki, G. Sugihara, M. Tanaka, and S. Kaneshina, J. Colloid Interface Sci. 74, 474 (1980). 
${ }^{19}$ M. Tanaka, S. Kaneshina, T. Tomida, K. Noda, and K. Aoki, J. Colloid Interface Sci. 44, 525 (1973).

${ }^{20}$ H. W. Offen, Rev. Phys. Chem. Jpn. 50, 97 (1980), available at http://hdl.handle.net/2433/47106.

${ }^{21}$ K. Hara, H. Kuwabara, O. Kajimoto, and K. Bhattacharyya, J. Photochem. Photobiol., A 124, 159 (1999).

${ }^{22}$ A. De Nicola, T. Kawakatsu, C. Rosano, M. Celino, M. Rocco, and G. Milano, J. Chem. Theory Comput. 11, 4959 (2015).

${ }^{23}$ D. Yordanova, I. Smirnova, and S. Jakobtorweihen, J. Chem. Theory Comput. 11, 2329 (2015).

${ }^{24}$ H. S. Muddana, H. H. Chiang, and P. J. Butler, Biophys. J. 102, 489 (2012).

${ }^{25}$ A. Pizzirusso, A. De Nicola, and G. Milano, J. Phys. Chem. B 120, 3821 (2016).

${ }^{26}$ P. S. Denkova, L. V. Lokeren, I. Verbruggen, and R. Willem, J. Phys. Chem. B 112, 10935 (2008).

${ }^{27}$ P. F. Fuchs, H. S. Hansen, P. H. Hünenberger, and B. A. Horta, J. Chem. Theory Comput. 8, 3943 (2012).

${ }^{28}$ M. P. Andersson and P. Uvdal, J. Phys. Chem. A 109, 2937 (2005).

${ }^{29}$ M. J. Frisch, G. W. Trucks, H. B. Schlegel, G. E. Scuseria, M. A. Robb, J. R. Cheeseman, J. A. Montgomery, Jr., T. Vreven, K. N. Kudin, J. C. Burant, J. M. Millam, S. S. Iyengar, J. Tomasi, V. Barone, B. Mennucci, M. Cossi, G. Scalmani, N. Rega, G. A. Petersson, H. Nakatsuji, M. Hada, M. Ehara, K. Toyota, R. Fukuda, J. Hasegawa, M. Ishida, T. Nakajima, Y. Honda, O. Kitao, H. Nakai, M. Klene, X. Li, J. E. Knox, H. P. Hratchian, J. B. Cross, V. Bakken, C. Adamo, J. Jaramillo, R. Gomperts, R. E. Stratmann, O. Yazyev, A. J. Austin, R. Cammi, C. Pomelli, J. W. Ochterski, P. Y. Ayala, K. Morokuma, G. A. Voth, P. Salvador, J. J. Dannenberg, V. G. Zakrzewski, S. Dapprich, A. D. Daniels, M. C. Strain, O. Farkas, D. K. Malick, A. D. Rabuck, K. Raghavachari, J. B. Foresman, J. V. Ortiz, Q. Cui, A. G. Baboul, S. Clifford, J. Cioslowski, B. B. Stefanov, G. Liu, A. Liashenko, P. Piskorz, I. Komaromi, R. L. Martin, D. J. Fox, T. Keith, M. A. Al-Laham, C. Y. Peng, A. Nanayakkara, M. Challacombe, P. M. W. Gill, B. Johnson, W. Chen, M. W. Wong, C. Gonzalez, and J. A. Pople, GaUssian 03, Revision C.02, Gaussian, Inc., Wallingford, CT, 2004.
${ }^{30}$ D. Van Der Spoel, E. Lindahl, B. Hess, G. Groenhof, A. E. Mark, and H. J. Berendsen, J. Comput. Chem. 26, 1701 (2005).

${ }^{31}$ B. Hess, C. Kutzner, D. Van Der Spoel, and E. Lindahl, J. Chem. Theory Comput. 4, 435 (2008).

${ }^{32}$ H. Berendsen, J. Grigera, and T. Straatsma, J. Phys. Chem. 91, 6269 (1987).

${ }^{33}$ G. Bussi, D. Donadio, and M. Parrinello, J. Chem. Phys. 126, 014101 (2007).

${ }^{34}$ H. J. Berendsen, J. P. M. Postma, W. F. van Gunsteren, A. DiNola, and J. Haak, J. Chem. Phys. 81, 3684 (1984).

${ }^{35}$ B. Hess, H. Bekker, H. J. Berendsen, and J. G. Fraaije, J. Comput. Chem. 18, 1463 (1997)

${ }^{36}$ M. J. Abraham and J. E. Gready, J. Comput. Chem. 32, 2031 (2011).

${ }^{37}$ D. Trzesniak, R. D. Lins, and W. F. van Gunsteren, Proteins: Struct., Funct., Bioinf. 65, 136 (2006).

${ }^{38}$ Y. R. Espinosa, J. R. Grigera, and E. R. Caffarena, Proteins: Struct., Funct., Bioinf. 85, 125 (2017).

${ }^{39}$ D. Yu, F. Huang, and H. Xu, Anal. Methods 4, 47 (2012).

${ }^{40}$ D. Linke, Methods Enzymol. 463, 603 (2009).

${ }^{41}$ X. Daura, K. Gademann, B. Jaun, D. Seebach, W. F. van Gunsteren, and A. E. Mark, Angew. Chem., Int. Ed. 38, 236 (1999).

${ }^{42}$ A. D. MacKerell, Jr., J. Phys. Chem. 99, 1846 (1995).

${ }^{43}$ C. D. Bruce, M. L. Berkowitz, L. Perera, and M. D. Forbes, J. Phys. Chem. B 106, 3788 (2002).

${ }^{44}$ K. Hara, H. Kuwabara, and O. Kajimoto, J. Phys. Chem. A 105, 7174 (2001).

${ }^{45}$ M. Lesemann, H. Nathan, T. DiNoia, C. Kirby, M. McHugh, J. Van Zanten, and M. Paulaitis, Ind. Eng. Chem. Res. 42, 6425 (2003).

${ }^{46}$ E. R. Caffarena and J. R. Grigera, Phys. A 342, 34 (2004).

${ }^{47}$ O. Chara, A. N. McCarthy, and J. R. Grigera, Phys. Lett. A 375, 572 (2011).

${ }^{48}$ S. Fanetti, A. Lapini, M. Pagliai, M. Citroni, M. Di Donato, S. Scandolo, R. Righini, and R. Bini, J. Phys. Chem. Lett. 5, 235 (2013).

${ }^{49}$ C. Rycroft, Chaos 19, 041111 (2009).

${ }^{50}$ S. Abel, F.-Y. Dupradeau, and M. Marchi, J. Chem. Theory Comput. 8, 4610 (2012).

${ }^{51}$ J. R. Errington and P. G. Debenedetti, Nature 409, 318 (2001). 